\title{
Formulación de dietas alimenticias para niños de la región Cajamarca, empleando programación lineal
}

\author{
Formulation of food diets for children in the Cajamarca region, using linear \\ programming \\ Lenin Quiñones $\mathrm{H}^{1}$ (i)* y María A Cueva $\mathrm{R}^{2}$ (1).
}

\begin{abstract}
RESUMEN
El 2019, la región de Cajamarca posee la segunda tasa más alta de desnutrición crónica en la población menor de cinco años de edad con un 27,4\%. En este sentido, el objetivo del trabajo fue formular dietas alimenticias para niños de la región Cajamarca, empleando programación lineal. La investigación fue descriptiva, consistió en aplicar el modelo matemático de dietas propuesta por Dantzing; para la formulación de las mismas se consideraron cincuenta y nueve (59) alimentos que se produce en la mencionada región, además se tomó en cuenta las recomendaciones dadas por la FAO con respecto a la cantidad de energía, proteínas y grasas que deben consumir los niños y finalmente se utilizó el software Octave para resolver el problema matemático. En el presente artículo se muestra la adaptación del modelo que permite formular dietas alimenticias (de costo mínimo) para niños de la región Cajamarca. Al emplear el modelo para determinar una adecuada dieta alimenticia que será consumida por un niño menor de cinco años; se obtuvo que el costo mínimo óptimo sería S/. 0.7, de esta manera garantiza los requerimientos nutricionales. Además, la cantidad que debería consumir 1,97 (49.7\%) de harina de plátano y $2.00064(50.4 \%)$ de harina de cacao.
\end{abstract}

Palabras clave: Malnutrición, pobreza, optimización, modelo matemático.

\begin{abstract}
In 2019, the region of Cajamarca has the second highest rate of chronic malnutrition in the population under five years of age with 27.4 percent. In this sense, the objective of the work was to formulate food diets for children in the Cajamarca region, using linear programming. The research was descriptive and consisted in applying the mathematical model of diets proposed by Dantzing; fifty-nine (59) foods produced in the region were considered for the formulation of the diets, and the recommendations given by the FAO regarding the amount of energy, protein and fat that children should consume were also taken into account, and finally the Octave software was used to solve the mathematical problem. This article shows the adaptation of the model that allows the formulation of (minimum cost) food diets for children in the Cajamarca region. Using the model to determine an adequate food diet to be consumed by a child under five years, it was obtained that the minimum optimal cost would be $\mathrm{S} / .0 .7$, thus ensuring the nutritional requirements. In addition, the amount that should consume $1.97(49.7 \%)$ of banana flour and 2,00064 (50.4\%) of cocoa flour.
\end{abstract}

Keywords: Malnutrition, poverty, optimization, mathematical model.

DOI: https://doi.org/10.37787/pakamuros-unj.v8i4.151

Recibido: 09/11/2020. Aceptado: 15/12/2020

* Autor para correspondencia

Universidad Nacional de Jaén, Perú. Email: lenin.quinones@unj.edu.pe

2. Universidad Nacional de Jaén, Perú. Email: maria_cueva@unj.edu.pe 


\section{INTRODUCCIÓN}

La alimentación es un factor que interviene en la salud de las personas, por consiguiente, si se elige, prepara e ingiere alimentos adecuados; estas acciones permitirían prevenir enfermedades. En consecuencia, actúa directamente en el desarrollo físico, psíquico e intelectual del niño (Echave Uranga, 2014). Es así que, se seleccionaron ingredientes y alimentos de bajo costo, disponibles localmente y familiares para la comunidad para formular complementos alimenticios para controlar enfermedades como la diabetes, la hipertensión y los trastornos. Los hallazgos generales revelaron que las mezclas funcionales de alimentos saludables reducen el riesgo de enfermedades coronarias, diabetes, hipertensión (Rajaram \& K, 2020).

La situación nutricional en América Latina y el Caribe es un indicador de las desigualdades sociales; igualmente, es causa y a su vez consecuencia de la pobreza. Mientras la producción de bienes e insumos alimentarios triplica los requerimientos energéticos de la población, 53 millones de personas tienen un acceso insuficiente a los alimentos. La región es en extremo heterogénea, con una gran diversidad de situaciones entre países y dentro de ellos (UNICEF, 2006). Perú, en el período 2000 al 2011 ha logrado disminuir sus tasas de desnutrición crónica, desnutrición aguda (DA) y de anemia. Las tasas de DA han descendido casi a un tercio, manteniéndose en cifras generales más bien bajas, y afecta en mayor medida a zonas y colectivos muy localizados del país. Sin embargo, las tasas de desnutrición crónica y de anemia siguen siendo altas (Sobrino et al., 2014). La desnutrición crónica infantil constituye uno de los principales problemas de Salud Pública en el país, según los valores de referencia de la OMS, la prevalencia nacional es del 19,5 \% en niños menores de cinco años (Sánchez-Abanto, 2012). Sin embargo, la región de Cajamarca posee la segunda tasa más alta de desnutrición crónica en la población menor de cinco años de edad con un 27,4 \% (INEI, 2019).

En los años 60 con la formulación del modelo de dieta basado en programación lineal; este sirvió como base para el software CAMP (Computer Assisted Menu Planning) desarrollado para computadoras. Durante las siguientes dos décadas, se introdujeron técnicas para incorporar las preferencias alimentarias en la planificación del menú. También, surgió el software para mini y microcomputadoras (Lancaster, 1992). Existen una variedad de softwares para la elaboración de mezclas alimenticias, sin embargo, no contienen en su base de datos productos andinos como el tarwi, cañihua, maca, quinua, maíz, kiwicha, o productos originales del área de estudio. Lo cual no permiten el uso de estos softwares comerciales para la formulación de mezclas alimenticias con cultivos andinos en el Perú por ejemplo; fue uno de los motivos para que el software "Formulación de Mezclas", se diseñe y la prueba unitaria de desarrolle (Carcasi, 2020). Existen varios métodos que se emplean para formular dietas balanceadas, 
entre ellos: aproximaciones sucesivas (prueba y error), ecuaciones simultáneas, cuadrado de Pearson, softwares comerciales y programación lineal (Campagna, s. f.).

La programación lineal ha dado a la humanidad la capacidad de establecer objetivos generales y de trazar un camino de decisiones detalladas a fin de lograr sus objetivos, cuando se enfrentan a situaciones prácticas de gran complejidad. Las herramientas para hacer esto, son opciones de formular problemas del mundo real en términos matemáticos (modelos), técnicas para resolver los modelos (algoritmos), y los motores para ejecutar los pasos de los algoritmos mediante computadoras y software (Dantzig \& Thapa, 2006). Es la técnica más empleada para la selección de mezclas de alimentos de menor costo, para cumplir con los requisitos nutricionales específicos para un grupo particular de personas, ya sea por razones de salud general o relacionadas con la enfermedad (Iwuji et al., 2016). En este sentido en Chile, se discriminó entre una serie de fuentes proteicas no convencionales alternativas, para la producción de una leche-cereal destinada al Programa de Alimentación Complementaria desarrollado en el mencionado país (Vega et al., 1995). Además, en Ecuador se ha empleado la teoría en mención, la cual ayudó a encontrar un costo mínimo los alimentos que permitan mantener o dar una mayor cantidad de nutrientes empleando el modelo (Rodriguez \& Molina Garzon, 2000). Puesto que, según las Naciones Unidas (ONU), los niños menores de 5 años que padecen desnutrición aguda severa, necesitan ser tratados con productos nutricionales especiales como los alimentos terapéuticos listos para usar (ATLC), de ahí que, se desarrolló un método basado en programación lineal y se puso a prueba en el diseño de un prototipo de ATLC para el tratamiento de la pérdida de peso involuntario de más del 10\% del peso corporal en niños y adultos de África oriental (Dibari et al., 2012).

Las razones, que han motivado realizar el presente estudio fueron cuatro: la primera, la región posee una gran riqueza, diversidad y disponibilidad de alimentos, pudiéndole dar valor agregado. Segunda, desde el punto de vista científico es relevante el empleo y la validación del modelo de dieta basado en programación lineal, en la formulación de dietas alimenticias para niños de la región Cajamarca. Tercera, desde el punto de vista funcional, al tener un modelo matemático confiable en tiempo real para formular dietas alimenticias, las instituciones del Estado y/o privados pueden emplearlo para su uso o proponer políticas alimentarias que permitan reducir la desnutrición crónica en la región Cajamarca, y finalmente, la cuarta es emplear modelos matemáticos, por razones de facilidad de implementación, con un costo relativamente inferior al de softwares comerciales.

En este contexto, el objetivo del trabajo fue formular dietas alimenticias de alto valor nutritivo para niños de la región Cajamarca, empleando programación lineal. 


\section{MATERIALES Y MÉTODOS}

\section{Tipo y diseño de la investigación}

El tipo de investigación fue descriptiva, puesto que los investigadores se han limitado a caracterizar situaciones, eventos y hechos que permitan adecuar un modelo matemático de dieta propuesto para formular dietas alimenticias para niños de la región Cajamarca. También, el diseño de la investigación fue no experimental, ya que no hubo manipulación de variables independientes.

\section{Método}

Deductivo y aplicativo, porque se analizaron las definiciones, propiedades, teoremas de programación lineal, el uso del software Octave y la aplicación del modelo matemático en la formulación de dietas alimenticias para niños de la región Cajamarca.

\section{Modelo matemático para formular dietas alimenticias}

El problema de la dieta en la programación lineal consiste en minimizar el costo total de los alimentos que serían empleados y asegurar que se cumplan algunas restricciones nutricionales. Smith realizó la primera solución al problema de la dieta empleando programación lineal. La función objetivo de estos problemas es la ecuación (1):

$$
\operatorname{Min} Z=C_{1} X_{1}+C_{2} X_{2}+C_{3} X_{3}+\cdots+C_{n} X_{n}
$$

Sujeta a las restricciones, ver ecuación (2) a la ecuación (6).

$$
\begin{gathered}
a_{11} X_{1}+a_{12} X_{2}+a_{13} X_{3}+\cdots+a_{1 n} X_{n}(\leq, \geq) b_{1} \\
a_{21} X_{1}+a_{22} X_{2}+a_{23} X_{3}+\cdots+a_{2 n} X_{n}(\leq, \geq) b_{2} \\
a_{31} X_{1}+a_{32} X_{2}+a_{33} X_{3}+\cdots+a_{3 n} X_{n}(\leq, \geq) b_{3} \\
a_{m 1} X_{1}+a_{m 2} X_{2}+a_{m 3} X_{3}+\cdots+a_{m n} X_{n}(\leq, \geq) b_{m} \\
X_{1}, X_{2}, X_{3}, \cdots+X_{n} \geq 0
\end{gathered}
$$

Donde:

$m$ es el número de nutrientes;

$n$ es el número de alimentos;

$a_{i j}$ es el número de unidades de nutrientes $i$ una unidad de alimento $j$;

$b_{i}$ es el número específico de unidades de nutrientes que necesito;

$C_{j}$ es el costo de los alimentos ítem $j$;

$X_{j}$ es el número de unidades de alimento ítem $j$ en la solución.

$i=1,2,3, \ldots, n$

$j=1,2,3, \ldots, m$

Para la búsqueda de la formulación óptima, se consideraron las restricciones del modelo de dieta, tomando en cuenta las recomendaciones FAO de 1981, ver Tabla 1. 
Tabla 1. Ingestas recomendadas de energía, proteínas y grasas

\begin{tabular}{lccc}
\hline Edad & $\begin{array}{c}\text { Energía } \\
\text { (Kcal/día) }\end{array}$ & $\begin{array}{c}\text { Proteínas } \\
\text { (g/día) }\end{array}$ & $\begin{array}{c}\text { Grasa total } \\
\text { (g/día) }\end{array}$ \\
\hline $4-8$ años & & & \\
\hline Niños & $1400-1700$ & 19 & $25-35$ \\
Niñas & $1300-1600$ & 19 & $25-35$ \\
\hline 9-13 años & & & \\
\hline Niños & $1800-2300$ & 34 & $25-35$ \\
Niñas & $1700-2000$ & 34 & $25-35$ \\
\hline
\end{tabular}

Fuente: FAO, 1951.

Los potenciales alimentos seleccionados para formular la dieta alimenticia, fueron cincuenta y nueve (59) alimentos producidos en la región de Cajamarca, Perú. Los precios, la composición de energía, proteína y grasa de los alimentos se muestran en la Tabla 2.

Tabla 2. Composición de energía, proteína y grasa de alimentos en Cajamarca

\begin{tabular}{|c|c|c|c|c|}
\hline Alimentos & Energía & Proteína & Grasa & Precio \\
\hline \multicolumn{5}{|l|}{ Cereales } \\
\hline Maíz amarillo & 355 & 6.7 & 4.8 & 0.10 \\
\hline Arroz blanco & 358 & 7.8 & 0.7 & 0.10 \\
\hline \multicolumn{5}{|l|}{ Alimentos procesados } \\
\hline Harina de plátano & 300 & 3.1 & 0.4 & 0.24 \\
\hline Harina de quinua & 343 & 13.6 & 5.8 & 0.36 \\
\hline Maní tostado & 590 & 27.1 & 51 & 0.20 \\
\hline Chancaca & 324 & 0 & 0 & 0.30 \\
\hline Avena hojuela cruda & 326 & 13.3 & 4 & 0.75 \\
\hline Yuca sancochada & 150 & 0.5 & 0.2 & 0.10 \\
\hline Maíz (jora seca) & 347 & 7 & 3.6 & 0.30 \\
\hline Harina de cacao & 404 & 19 & 17.1 & 0.12 \\
\hline \multicolumn{5}{|l|}{ Frutas } \\
\hline Aguaje & 283 & 2.3 & 25.1 & 0.30 \\
\hline Coco & 286 & 3.4 & 28.1 & 0.20 \\
\hline Cocona & 41 & 0.7 & 0.8 & 0.35 \\
\hline Granadilla & 80 & 2.2 & 2 & 0.35 \\
\hline Guanábana & 56 & 0.9 & 0.2 & 0.35 \\
\hline Guayaba & 56 & 0.5 & 0.01 & 0.10 \\
\hline Lima & 27 & 0.6 & 0.4 & 0.20 \\
\hline Lúcuma & 99 & 1.5 & 0.8 & 0.35 \\
\hline Mamey & 37 & 0.5 & 0.1 & 0.30 \\
\hline Maracuyá & 67 & 0.9 & 0.1 & 0.30 \\
\hline
\end{tabular}




\begin{tabular}{|c|c|c|c|c|}
\hline Níspero & 47 & 0.3 & 0 & 0.30 \\
\hline Guaba & 56 & 0.6 & 0.1 & 0.50 \\
\hline Pan de Árbol & 135 & 4.5 & 1.8 & 0.25 \\
\hline Piña & 38 & 0.4 & 0.2 & 0.35 \\
\hline Plátano de seda & 83 & 1.5 & 0.3 & 0.20 \\
\hline Plátano guineo & 120 & 1.4 & 0.2 & 0.20 \\
\hline Plátano manzana & 90 & 1.1 & 0.2 & 0.20 \\
\hline Plátano verde sancochado & 137 & 0.7 & 0.3 & 0.20 \\
\hline Tamarindo & 239 & 2.8 & 0.6 & 0.30 \\
\hline Mango ciruelo & 56 & 0.6 & 0.3 & 0.10 \\
\hline Tumbo & 64 & 1.2 & 0.5 & 0.10 \\
\hline Carambola & 35 & 1 & 0.6 & 0.10 \\
\hline Tomate de árbol & 43 & 1.6 & 0.2 & 0.10 \\
\hline \multicolumn{5}{|l|}{ Pescados } \\
\hline Bagre & 80 & 15.2 & 1.7 & 0.90 \\
\hline Boquichico & 273 & 47.9 & 9.1 & 0.90 \\
\hline Carachama & 64 & 14.2 & 0.4 & 0.85 \\
\hline Trucha blanca & 113 & 19.5 & 3.1 & 0.90 \\
\hline \multicolumn{5}{|l|}{ Animales menores } \\
\hline Cerdo & 198 & 14.4 & 15.1 & 2.50 \\
\hline Chivo & 115 & 19.4 & 3.6 & 2.00 \\
\hline Gallina & 108 & 19.2 & 2.9 & 2.00 \\
\hline Pato & 326 & 16 & 28.6 & 1.90 \\
\hline Pavo & 160 & 20.4 & 8 & 2.50 \\
\hline Cuy & 96 & 19 & 1.6 & 1.90 \\
\hline Pollo & 119 & 21.4 & 3.1 & 0.70 \\
\hline \multicolumn{5}{|l|}{ Animales mayores } \\
\hline Res & 105 & 21.4 & 3.1 & 2.20 \\
\hline \multicolumn{5}{|l|}{ Otras fuentes de proteína } \\
\hline Leche fresca & 63 & 3.1 & 3.5 & 0.30 \\
\hline Huevo & 139 & 12.9 & 8.4 & 0.30 \\
\hline Café & 2 & 0.1 & 0 & 1.00 \\
\hline Hormiga ziki zapa & 486 & 36.3 & 28.9 & 0.40 \\
\hline \multicolumn{5}{|l|}{ Tubérculos y raíces } \\
\hline Yuca & 168 & 1.5 & 0.2 & 0.20 \\
\hline Arracacha & 97 & 0.7 & 0.3 & 0.30 \\
\hline Yacon & 54 & 0.3 & 0.3 & 0.30 \\
\hline Sachapapa & 141 & 2.7 & 0.1 & 0.50 \\
\hline Pituca & 102 & 8.1 & 0.3 & 0.20 \\
\hline \multicolumn{5}{|l|}{ Leguminosas } \\
\hline Frejol amarillo & 334 & 21.1 & 1.5 & 0.25 \\
\hline Frejol bayo/negro & 333 & 20.3 & 1.5 & 0.25 \\
\hline Frejol soya & 401 & 28.2 & 18.9 & 0.40 \\
\hline
\end{tabular}




\begin{tabular}{lllll} 
Pallares & 331 & 20.4 & 1.2 & 0.25 \\
Lentejas & 338 & 23.4 & 1.1 & 0.25 \\
\hline
\end{tabular}

Fuente: Instituto Nacional de Salud.

\section{Herramienta computacional}

Para resolver el problema matemático, programación lineal o procedimiento de optimización, se utilizaron las funciones implementadas en el software GNU Octave, versión 5.1.0.

\section{RESULTADOS}

El modelo de dieta adecuado, se ilustra utilizando tres alimentos: harina de quinua, harina de plátano y harina de cacao. Para este fin, se ha considerado el plan de dieta óptima para un día que debe ingerir un niño en edad escolar menor de 5 años, considerando la necesidad calórica recomendada por la FAO y la composición nutricional de los tres alimentos, ver Tabla 3.

Tabla 3. Composición química de harina de quinua, harina de plátano y harina de cacao.

\begin{tabular}{lcccc}
\hline & $\begin{array}{c}\text { Harina de quinua } \\
(\mathbf{g} / \mathbf{1 0 0 g} \text { de alimento) }\end{array}$ & $\begin{array}{c}\text { Harina de } \\
\text { Plátano }(\mathbf{g} / \mathbf{1 0 0 g} \\
\text { de alimento) }\end{array}$ & $\begin{array}{l}\text { Harina de cacao } \\
\text { (g/100g de } \\
\text { alimento })\end{array}$ & $\begin{array}{c}\text { Requerimientos } \\
\text { nutricionales g/día }\end{array}$ \\
\hline Energía & 343 & 300 & 404 & $1400-1700 \mathrm{kcal}$ \\
Proteína & 13,6 & 3,1 & 19 & 19 \\
Grasa & 5,8 & 0,4 & 17.1 & $25-35$ \\
Costo & 0.36 & 0.24 & 0.12 & \\
\hline
\end{tabular}

Las variables de decisión fueron:

$x_{1}$ : Cien gramos de harina de quinua utilizados en la dieta alimenticia

$x_{2}$ : Cien gramos de harina de plátano utilizada en la dieta alimenticia

$x_{3}$ : Cien gramos de harina de cacao utilizadas en la dieta alimenticia

La función objetivo fue minimizar los costos de la dieta alimentaria para niños menor de cinco años, ecuación (7).

$$
\operatorname{Min} \mathrm{Z}=0.36 x_{1}+0.24 x_{2}+0.12 x_{3}
$$

Las restricciones, que permiten satisfacer los requerimientos nutricionales para niños menores de cinco años, ver ecuación (8) hasta ecuación (13).

Energía:

Proteína:

$$
\begin{aligned}
& 343 x_{1}+300 x_{2}+404 x_{3} \geq 1400 \\
& 343 x_{1}+300 x_{2}+404 x_{3} \leq 1700
\end{aligned}
$$


Grasa:

$$
13,6 x_{1}+3,1 x_{2}+19 x_{3} \geq 19
$$

$$
\begin{aligned}
& 5,8 x_{1}+0,4 x_{2}+17,1 x_{3} \geq 25 \\
& 5,8 x_{1}+0,4 x_{2}+17,1 x_{3} \leq 35
\end{aligned}
$$

No negatividad:

$$
x_{1} \geq 0, x_{2} \geq 0, x_{3} \geq 0
$$

El problema de programación lineal planteado, se ha resuelto empleando el software Octave, ver Figura 1. Del cuál, todas las posibles cantidades al hacer las combinaciones de los tres alimentos. El costo mínimo óptimo sería S/. 0.7, de esta manera garantiza los requerimientos nutricionales. Además, la cantidad que debería consumir 1,97 (49.7\%) de harina de plátano, 2.00064 (50.4 \%) de harina de cacao y $0(0 \%)$ de harina de quinua.

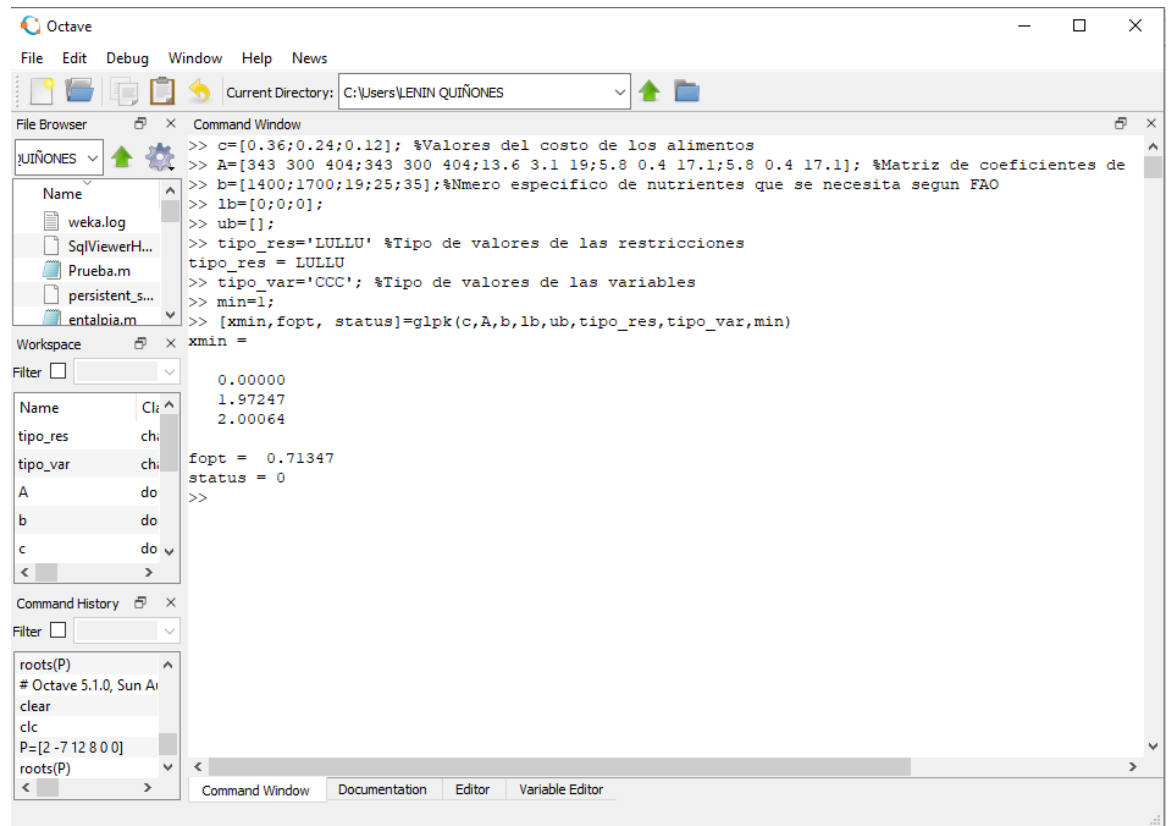

Figura 1. Solución del modelo matemático empleando el software Octave.

\section{DISCUSIÓN}

La presente investigación fue desarrollada para la región Cajamarca, debido que ocupa el segundo lugar en desnutrición crónica y anemia en la población infantil menor de cinco años. Pero es aplicable a las demás regiones; que tengan como objetivo formular mezclas alimenticias con productos de su zona. Del conjunto de materias primas (cincuenta y nueve), consideradas por su disponibilidad y hábitos de consumo en la región Cajamarca. Para efectos demostrativos del empleo del modelo, se tomaron en consideración tres (harina de plátano, harina de cacao y harina de quinua). Se solucionó el problema planteado, originándose un valor óptimo de costo accesible al consumidor. Encontrándose una solución factible y el óptimo correspondió a la distribución de ingredientes: $49.7 \%$ de harina de plátano, 50.4\% de harina de cacao y $0 \%$ de harina de quinua. Donde, el costo mínimo óptimo sería de S/ 0.70 por la 
compra de los alimentos que compondrían la dieta alimentaria, de esta manera si el niño las ingiriera estaría cumpliendo con los requerimientos concerniente a energía, proteína y grasa, dadas por la FAO, ver Tabla 1. Además, si se establecen modificaciones al problema original, es posible generar nuevas soluciones, con un pequeño incremento del costo de la formulación.

Basándose en resultados sobre valor nutricional de las tres materias primas: La harina de quinua, es rica en proteínas; tiene varios competidores nutricionales como la harina de cacao con respecto a aporte en lípidos y proteínas de alta calidad funcional por ser de origen vegetal. Por otro lado, la harina de plátano posee carbohidratos, fibras, vitaminas y minerales que no contienen la harina de quinua y la harina de cacao. Además, la harina de cacao posee grasas saludables, proteínas, antioxidantes. En consecuencia, los resultados del modelo son la combinación óptima tanto de nutrientes como en el precio de adquisición de los mismos.

El trabajo, confirma que el modelo de dieta alimenticia se puede emplear para requisitos nutricionales específicos para un grupo particular de personas, ya sea por razones de salud general o relacionadas con la enfermedad (Iwuji et al., 2016). Corroborando lo mencionado en el trabajo (Bohrer, 2019), que los productos cárnicos análogos modernos pueden ofrecer aproximadamente la misma composición de nutrientes que los productos cárnicos tradicionales. Al igual que el trabajo de (Carcasi, 2020), se pueden incluir en su base de datos del programa productos originales de la región de Cajamarca.

Las limitaciones de los resultados fueron: El escaso empleo de modelos matemáticos para determinar las dietas alimentarias para niños de la región Cajamarca; debido, a que estos utilizan programas especializados o teorías matemáticas de nivel universitario que no está al alcance cognoscitivo de los usuarios. El estudio no fue experimental, lo cual no permitió aplicar la formulación con la propuesta de tres materias primas, tal como se propuso en (Aro Aro \& Calsin Cutimbo, 2019).

Es un trabajo multidisciplinario, dirigida a una población vulnerable como son los niños menores de cinco años. Donde, la información obtenida en este trabajo permite desarrollar procesos de laboratorio adecuados para hacer realidad las formulaciones propuestas. De manera análoga, donde se empleó un método basado en programación lineal para el tratamiento de la pérdida de peso involuntario de más del $10 \%$ del peso corporal en niños y adultos de África oriental (Dibari et al., 2012). Incluso se puede discriminar entre las cincuenta y nueve materias primas, para la diversificación de productos destinada a la disminución de brecha de desnutrición crónica mediante productos organolépticos aceptables para el público objetivo. Mencionar que en Chile desarrollaron una leche - cereal para el Programa de Alimentación Complementaria (Vega et al., 1995). 


\section{CONCLUSIONES}

Se adecúo el modelo para formular dietas alimenticias (de costo mínimo) para niños de la región Cajamarca. Posteriormente se aplicó el instrumento para un niño menor de cinco años, se obtuvo que el costo mínimo óptimo sería S/. 0.7, garantizando los requerimientos nutricionales dados por la FAO. Además, la cantidad que debería consumir $49.7 \%$ de harina de plátano y $50.4 \%$ de harina de cacao.

Finalmente, se incluye algunas líneas futuro de trabajo derivado del desarrollo logrado: (a) Realizar una aplicación móvil que permita formular las dietas alimenticias para los niños de la Región Cajamarca; considerando alimentos que produce la región. (b) Posteriormente realizar estudios experimentales, sobre el empleo y comportamiento de la herramienta tecnológica, en las provincias de Cajamarca con alto índice de desnutrición crónica. (c) Desarrollar algoritmos basados en minería de datos, programación lineal difusa, etc. para poder comparar con los resultados de algoritmos clásicos y obtener mejores resultados de predicción.

\section{REFERENCIAS BIBLIOGRÁFICAS}

Aro Aro, J. M., \& Calsin Cutimbo, M. (2019). Elaboración de una mezcla alimenticia a base de quinua (Chenopodium quinoa Willd), cañihua (Chenopodium pallidicaule Aellen), cebada (Hordeum vulgare L.) maiz (Zea mays L.), haba (Vicia faba L.) y soya (Glycine max L. Merr) por proceso de cocción-Extrusión. Revista de Investigaciones Altoandinas, 21(4), 293-303. https://doi.org/10.18271/ria.2019.506

Bohrer, B. M. (2019). An investigation of the formulation and nutritional composition of modern meat analogue products. Food Science and Human Wellness, 8(4), 320-329. https://doi.org/10.1016/j.fshw.2019.11.006

Campagna, D. (s. f.). Formulación de dietas. Centro de Información de Actividades Porcinas.

Carcasi, P. A. (2020). Diseño y análisis de un software para formulación de mezclas alimenticias a base de cultivos andinos: Revista Colombiana de Investigaciones Agroindustriales, 7(1), 30-41. https://doi.org/10.23850/24220582.2571

Dantzig, G. B., \& Thapa, M. N. (2006). Linear programming. Operations Research, 50(1), 42-47.

Dibari, F., Diop, E. H. I., Collins, S., \& Seal, A. (2012). Low-cost, ready-to-use therapeutic foods can be designed using locally available commodities with the aid of linear programming. The Journal of nutrition, 142(5), 955-961.

Echave Uranga, L. M. (2014). La importancia de la alimentación en la edad infantil. Revista Arista Digital, 49, 57-61. 
INEI. (2019). Desnutrición crónica afectó al 12,2\% de la población menor de cinco años de edad en el año 2018. Instituto Nacional de Estadística e Informática. https://www.inei.gob.pe/prensa/noticias/desnutricion-cronica-afecto-al-122-de-la-poblacionmenor-de-cinco-anos-de-edad-en-el-ano-2018-11370/

Iwuji, A. C., Nnanna, M., \& Ndulue, N. I. C. (2016). An optimal DASH diet model for people with hypertension using linear programming approach. Open Journal of Optimization, 5(01), 14.

Lancaster, L. M. (1992). The evolution of the diet model in managing food systems. Interfaces, 22(5), 59-68.

Rajaram, U., \& K, L. U. (2020). Formulation and Evaluation of Functional food Health mixes. International Journal of Research in Pharmaceutical Sciences, 11(3), 4347-4352. https://doi.org/10.26452/ijrps.v11i3.2651

Rodriguez, L., \& Molina Garzon, G. G. (2000). Banco de datos y modelo matematico para el cálculo de dietas familiares [Tesis de pre grado, Escuela Superior Politécnica del Litoral]. http://www.dspace.espol.edu.ec/handle/123456789/4171

Sánchez-Abanto, J. (2012). Evolución de la desnutrición crónica en menores de cinco años en el Perú. Revista Peruana de Medicina Experimental y Salud Publica, 29(3), 402-405. http://www.scielo.org.pe/scielo.php?script=sci_abstract\&pid=S172646342012000300018\&lng=es\&nrm=iso\&tlng=es

Sobrino, M., Gutiérrez, C., Cunha, A. J., Dávila, M., \& Alarcón, J. (2014). Desnutrición infantil en menores de cinco años en Perú: Tendencias y factores determinantes. Revista Panamericana de Salud Pública, 35, 104-112. https://www.scielosp.org/article/rpsp/2014.v35n2/104-112/

UNICEF. (2006). Desnutrición infantil en América Latina y el Caribe. Desafíos, 2, 5-9.

Vega, R., Fagetti, E., Gaete, F., \& Romo, C. (1995). Desarrollo de una leche-cereal. I. elección de ingredientes por programación lineal. Contribuciones Científicas y Tecnológicas, 95, 27-38. http://www.revistas.usach.cl/ojs/index.php/contribuciones/article/view/2111 